\title{
Taking into account the temperament factor: clinical and therapeutic implications Konstantinos N Fountoulakis
}

\author{
Address: 3rd Department of Psychiatry, Aristotle University of Thessaloniki, Greece \\ from International Society on Brain and Behaviour: 3rd International Congress on Brain and Behaviour \\ Thessaloniki, Greece. 28 November - 2 December 2007 \\ Published: 17 April 2008 \\ Annals of General Psychiatry 2008, 7(Suppl I):S8I doi:I0.II86/I744-859X-7-SI-S8I
}

This abstract is available from: http://www.annals-general-psychiatry.com/content/7/SI/S8I

(C) 2008 Fountoulakis; licensee BioMed Central Ltd.

Temperament, although an ancient concept, seems to go far beyond modern classification systems and utilizes a wealth of valuable information including personal and family history as well as 'non-typical' symptoms. Thus, it succeeds to define both the specific features of the individual mood episode as well as the long term outcome of the disease, and particularly the response to treatment and overall outcome. The assessment of the temperament is not typically utilized when the therapist designs the treatment strategy. The usual practice is to prescribe antidepressants for depressive episodes and antipsychotics for psychotic symptoms; specific antiepileptics and lithium can be used as mood stablilizers. According to this practice, the 'classic' clinical pictures are easier to recognize and to treat, and most agents preferably treat with higher rates of success specific clinical entities, for example lithium is more useful for bipolar patients with euphoric mania and antidepressants as monotherapy for unipolar melancholic depression. However, today, the majority of patients do not fall into these 'classic' categories and the assessment of the temperament can reveal a number of clues highly predicting response to a specific treatment as well as the manifestation of a number of problematic effects of pharmacotherapy like agitation, induction of mixed states, rapid cycling and suicidality and even weight gain. Moreover, the knowledge of the temperament can guide the design of the therapeutic strategy with informed and careful simultaneous use of multiple agents. This strategy among others should include some but not all second generation antipsychotics aiming not to sedate, but to the fast acute treatment and prophylaxis from excessive hyperthymic or overt manic behaviours as well as suicide. 